\title{
fMRI Syntactic and Lexical Repetition Effects Reveal the Initial Stages of Learning a New Language
}

\author{
○Kirsten Weber, ${ }^{1,2}$ Morten H. Christiansen, ${ }^{3}$ Karl Magnus Petersson, ${ }^{1}$ Peter Indefrey, ${ }^{4}$ and $®$ Peter Hagoort ${ }^{1,2}$ \\ ${ }^{1}$ Max Planck Institute for Psycholinguistics, 6526 XD Nijmegen, The Netherlands, ${ }^{2}$ Donders Institute for Brain, Cognition and Behaviour, Donders Centre \\ for Cognitive Neuroimaging, Radboud University Nijmegen, 6525 EN Nijmegen, The Netherlands, ${ }^{3}$ Department of Psychology, Cornell University, Ithaca, \\ New York 14853, and ${ }^{4}$ Department of Linguistics, Heinrich-Heine-University Düsseldorf, 40225 Düsseldorf, Germany
}

When learning a new language, we build brain networks to process and represent the acquired words and syntax and integrate these with existing language representations. It is an open question whether the same or different neural mechanisms are involved in learning and processing a novel language compared with the native language(s). Here we investigated the neural repetition effects of repeating known and novel word orders while human subjects were in the early stages of learning a new language. Combining a miniature language with a syntactic priming paradigm, we examined the neural correlates of language learning on-line using functional magnetic resonance imaging. In left inferior frontal gyrus and posterior temporal cortex, the repetition of novel syntactic structures led to repetition enhancement, whereas repetition of known structures resulted in repetition suppression. Additional verb repetition led to an increase in the syntactic repetition enhancement effect in language-related brain regions. Similarly, the repetition of verbs led to repetition enhancement effects in areas related to lexical and semantic processing, an effect that continued to increase in a subset of these regions. Repetition enhancement might reflect a mechanism to build and strengthen a neural network to process novel syntactic structures and lexical items. By contrast, the observed repetition suppression points to overlapping neural mechanisms for native and new language constructions when these have sufficient structural similarities.

Key words: fMRI; language learning; miniature language; priming; repetition effects; syntax

\section{Significance Statement}

Acquiring a second language entails learning how to interpret novel words and relations between words, and to integrate them with existing language knowledge. To investigate the brain mechanisms involved in this particularly human skill, we combined an artificial language learning task with a syntactic repetition paradigm. We show that the repetition of novel syntactic structures, as well as words in contexts, leads to repetition enhancement, whereas repetition of known structures results in repetition suppression. We thus propose that repetition enhancement might reflect a brain mechanism to build and strengthen a neural network to process novel syntactic regularities and novel words. Importantly, the results also indicate an overlap in neural mechanisms for native and new language constructions with sufficient structural similarities.

\section{Introduction}

Learning a new language is a formidable feat for which we have to develop a complex set of linguistic skills, including encoding the words of the new language, learning syntactic structure, and in-

Received Aug. 24, 2015; revised May 10, 2016; accepted May 14, 2016.

Author contributions: K.W., M.H.C., K.M.P., P.I., and P.H. designed research; K.W. performed research; K.W. analyzed data; K.W., M.H.C., K.M.P., P.I., and P.H. wrote the paper.

This work was supported by a Toptalent PhD Grant from the NWO (Dutch Science Foundation), Grant 021.001.007. During the revisions of this paper, K.W. was supported by a fellowship from the Hanse Institute for Advanced Studies.

The authors declare no competing financial interests.

Correspondence should be addressed to Dr. Kirsten Weber, Max Planck Institute for Psycholinguistics, PO Box 310, 6500 AH Nijmegen, the Netherlands. E-mail: kirsten.weber@mpi.nl.

DOI:10.1523/JNEUROSCI.3180-15.2016

Copyright $\odot 2016$ the authors $\quad 0270-6474 / 16 / 366872-09 \$ 15.00 / 0$ tegrating the resulting representations with existing language knowledge. Here we used an fMRI repetition paradigm (Henson and Rugg, 2003) to investigate how adult learners acquire syntactic structures and words in the context of a miniature language.

In neuroimaging experiments, there is a contrast between repetition effects to known items (from objects to words to syntactic structures), which results in a reduction in activation: repetition suppression (RS), and repetition effects to novel items (eg, unknown objects, pseudowords), where repetition is accompanied by an increase in activation (Henson et al., 2000; Gagnepain et al., 2008): repetition enhancement (RE). Whereas RS is thought to reflect the facilitation of processing within or the sharpening of an existing neural representation, RE in the context of novel item repetition has been linked to the formation of neural representations (Grill-Spector et al., 2006; Segaert et al., 2013). 
Table 1. Example of one of the lists of lexical items

\begin{tabular}{|c|c|c|c|c|c|}
\hline \multicolumn{2}{|c|}{ Transitive verbs } & \multicolumn{2}{|c|}{ Transitive verbs } & \multicolumn{2}{|c|}{ Intransitive verbs } \\
\hline Alienese & English (Dutch) & Alienese & English (Dutch) & Alienese & English (Dutch) \\
\hline Basi & To dress (aankleden) & Agero & To shoot (neerschieten) & Atoku & To dry (afdrogen) \\
\hline Dase & To chase (achtervolgen) & Epaki & To topple s.o. (omtrekken) & Mikuro & To yawn (gapen) \\
\hline Haki & To dry s.o. (afdrogen) & Hakaro & To pick s.o. up (optillen) & Parube & To bend over (buigen) \\
\hline Kisu & To scare (bangmaken) & Hakenu & To help getting up (overeindhelpen) & Simera & To dance (dansen) \\
\hline Momu & To serve (bedienen) & Hakoba & To annoy (pesten) & Tokasi & To think (denken) \\
\hline Mose & To hassle (bedreigen) & Hipare & To kick (schoppen) & Mimu & To jump (hinkelen) \\
\hline Nago & To greet (begroeten) & Imera & To hit (slaan) & Ote & To cry (huilen) \\
\hline Nosoku & To pay (betalen) & Misabe & To tow (slepen) & Suki & To clap (klappen) \\
\hline Nuga & To jostle (duwen) & Mukare & To stop (stoppen) & Ucha & To beckon (zwaaien) \\
\hline Oku & To photograph (fotograferen) & Nagabi & To draw (tekenen) & Ugo & To drink (drinken) \\
\hline Omo & To help (helpen) & Nurasi & To console (troosten) & Nouns & \\
\hline Ona & To interview (interviewen) & Odaku & To wave goodbye (uitzwaaien) & Josa & Woman (vrouw) \\
\hline Sawe & To hug (knuffelen) & Odoka & To tie someone (vastbinden) & Komi & Man (man) \\
\hline Sitagu & To massage (masseren) & Odosi & To attend to s.o. (verzorgen) & Sako & Boy (jongen) \\
\hline Sosa & To tow (meetrekken) & Osuta & To find (vinden) & Miru & Girl (meisje) \\
\hline Teso & To measure (meten) & Sikimo & To feed (voeren) & & \\
\hline Tomi & To call after (naroepen) & Utape & To send away (wegsturen) & & \\
\hline Tose & To make wet (natmaken) & Utuso & To choke (wurgen) & & \\
\hline
\end{tabular}

There were eight such lists with different Alienese-to-English meaning mapping.

Behaviorally, syntactic repetition effects are well studied (Ferreira and Bock, 2006). The implicit learning theory sees syntactic priming as a mechanism for language learning (Chang et al., 2000) as the repetition of syntactic structures helps in mapping meaning onto form. Thus, syntactic priming effects might be present within the first hours of language learning. Furthermore, infrequent structures should benefit most from the repetition of structure ("inverse preference"), as their representations can be strengthened the most (Ferreira and Bock, 2006). There is also evidence for a lexical boost to syntactic priming (Tooley and Bock, 2014). Consequently, the residual activation account (Pickering and Branigan, 1998) links syntactic processing to the activation of syntactic frames in the mental lexicon in line with theories of syntactic processing (Vosse and Kempen, 2000; Jackendoff, 2002) that put the major part of syntactic information in the lexicon. During learning, verb repetition might help in boosting the mapping between form and meaning.

Neural processing of syntax activates a core network of left inferior frontal gyrus (LIFG) and left posterior middle and superior temporal gyrus (MTG/STG; Snijders et al., 2009; Segaert et al., 2012; Friederici and Gierhan, 2013). The LIFG has been linked to grammatical regularities in miniature languages and artificial grammars (Opitz and Friederici, 2004; Petersson et al., 2004; Petersson and Hagoort, 2012); the left posterior MTG/STG on the other has been linked to lexically driven grammatical knowledge (Hagoort, 2005).

In the current study participants learned a miniature language with two novel word orders and a third from their native language; the language included 46 novel verbs. The syntactic regularities and the mapping of structure and lexical items onto meaning had to be learned from the language input and the context without explicit instruction. To assess syntactic and lexical learning and processing effects, we used fMRI repetition effects to repeated presentations of syntactic structures (word orders) and lexical items (verbs). We predicted that repetition of novel word orders and words would lead to RE, as a new neural network for processing these structures and lexical items has to be created. Over days, while a new representation for the novel learned information is being built, the RE effects should continue to increase, whereas, once a representation is established, sharpening and facilitatory processes induced by the repetition should lead to RS. A similar logic should hold for the novel lexical items. If the
RE effects are linked to learning they should also correlate with the behavioral learning outcome. In contrast, a known syntactic structure that can be mapped onto a familiar word order should show RS early on. Moreover, considering the inverse preference account of syntactic priming, we expect the largest RE effect to infrequent structures. To investigate lexically driven syntactic learning we manipulated syntactic and verb repetition orthogonally to investigate whether the combined repetition of word order and verb would boost the syntactic repetition effects.

\section{Materials and Methods}

\section{Participants}

Twenty right-handed Dutch native speakers ( 16 female, 4 male) participated in this study, all with normal or corrected to normal vision and no history of neurological or language impairments (5 additional participants did not complete the full experiment and were therefore excluded from the data analysis). The participants received course credits or money for their participation in the experiment and all participants gave written informed consent.

\section{Materials}

The artificial language consisted of 36 transitive verbs, 10 intransitive verbs, and 4 nouns (Table 1). There were four different types of sentence structure in this language (Fig. 1a,b). Two were novel transitive word orders that are not permissible for Dutch transitive sentences: verbobject-subject (VOS) and object-subject-verb (OSV); a third transitive word-order was subject-verb-object (SVO), the "active" word order in Dutch, and thus known to the participants; the fourth sentence structure was an intransitive subject-verb (SV) word order, also present in Dutch, which was used in filler sentences. All subjects and objects were animate (man, woman, girl, boy). Lexical items were novel with an easy to produce syllabic structure (Table 1). A list of lexical items was rated by six Dutch native speakers and those that resembled Dutch or otherwise meaningful words were removed. The assignment of meaning to the different words and the word order of the frequent and infrequent novel structure were counterbalanced across subjects. The sentences described events depicted in black and white photographs (taken from a previous study; Menenti et al., 2011). There were eight possible depictions of each event. These were realized using two sets of actor pairs (girl/boy and woman/man), where the agent was either the male or the female actor and was located either to the left or to the right in the picture.

\section{Experimental procedure}

Participants took part in the experiment on four different days, Days $1,2,3$, and 9 (the latter could vary between Days 7 and 10). They were 
A Trial structure and presentation details

prime
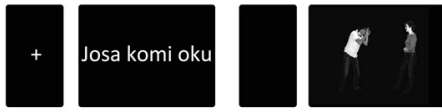

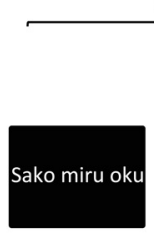

target

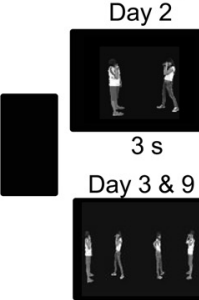

$(13)^{\star}$ filler
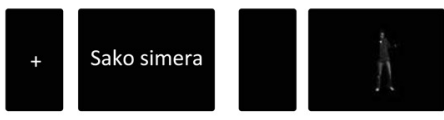

Button press $\mathrm{L}$ or $\mathrm{R}$

2s jitter

$3 s$

B Factors and conditions

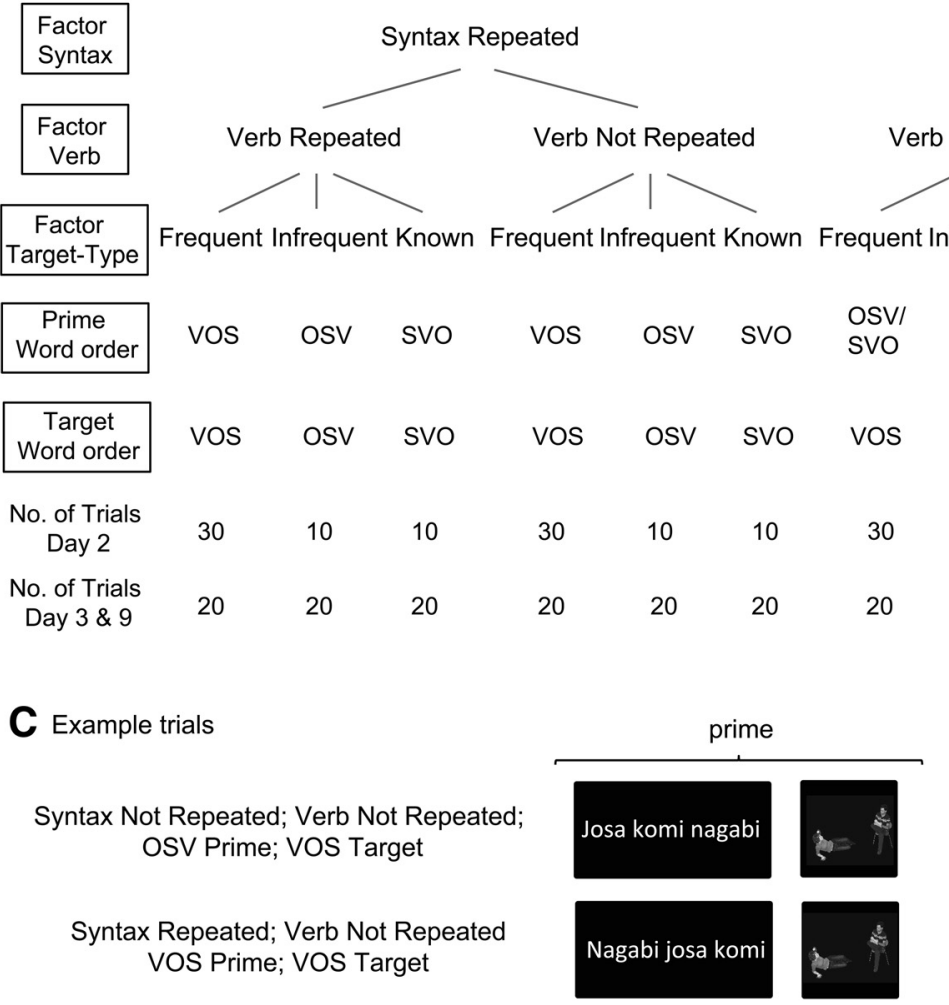

Syntax Not Repeated

Verb Repeated Verb Not Repeated

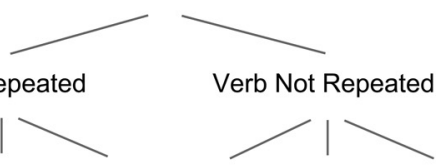

$>1>$

(n)

VOSI OSVI OSVI VOSI OSVI

SVO VOS SVO SVO VOS

osv SVO VOS OSV SVO

$\begin{array}{lllll}10 & 10 & 30 & 10 & 10 \\ 20 & 20 & 20 & 20 & 20\end{array}$

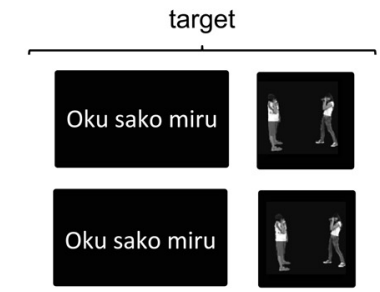

Figure 1. Trial structure and experimental conditions. $A$, Trial structure of a prime-target pair (both OSV word order in this example) followed by a filler trial (SV word order). $0 \mathrm{n}$ Day 2 the target trial sentence would be followed by a matching picture, on Days 3 and 9 participants would have to choose between two pictures showing the same action with the roles reversed. $\boldsymbol{B}$, Illustration of the different factors and conditions. One of the two possible word order to target structure-type mapping is shown (the other is frequent: 0SV; infrequent: VOS; known: SVO; counterbalanced across participants). The frequency manipulation was introduced on Day 2 (see number of trials Day 2). On Days 3 and 9 all target types occurred equally often. $\boldsymbol{C}$, Two examples of possible prime-target pairs.

told that they were going to learn a new language, "Alienese." On Day 1 , structural and functional MRI data were acquired. In a short functional session, sentences from the language they were about to learn were visually presented. This condition served as a baseline for the analysis. Subsequently, participants learned the four nouns outside the scanner, the words for man, woman, boy, and girl by means of a picture-word matching paradigm. First, each word was given with a matching picture six times, all nouns intermixed. To verify the learning, the pictures were then given with the four possible nouns. Participants had to choose the matching noun by a button press. Participants had learned all four nouns by the end of the experiment (after 6 more repetitions of each noun).

On Days 2, 3, and 9, participants took part in language learning sessions in the fMRI scanner in which they read sentences in the new language and saw pictures describing these. Unbeknownst to the participants, underlying these sessions was a repetition paradigm on the experimental items (Fig. 1). On Day 2, 80\% of a total of 300 sentences were experimental items and 20\% were filler sentences (intransitives). All in all, including filler sentences, word-order 1 (counterbalanced across participants between VOS and OSV) occurred $40 \%$ of the time and the other three word orders (word-order 2, known word-order, and intransitive word-order) $20 \%$ of the time. Participants were asked to read the sentences silently. After each sentence a picture was displayed illustrating its meaning (Fig. 1a,b). In subsequent experimental items, verbs, and word orders were repeated in one-half of the cases, orthogonally to each other (25\% verbs only repeated, $25 \%$ syntax only repeated, $25 \%$ both repeated, $25 \%$ neither repeated). The nouns were never repeated in subsequent sentences, ie, sentences containing the woman and the man alternated with those containing the boy and the girl. One to three filler items with an SV sentence structure (Fig. $1 a$; last item for an SV example) were interspersed between the experimental trials. The priming setup was thus not continuous; a target did not serve as the immediate prime of the next trial. The procedure on Days 3 and 9 was similar to the one on Day 2, except 
that all word orders occurred equally often, with 20 trials per condition. In addition to reading the sentences, the subjects now had to perform a comprehension task. After each target sentence, the participants were presented with two pictures (Fig. 1a). Both pictures depicted the same action with the same actors, but the roles of the actors (agent and patient) were reversed. Participants were asked to decide which picture matched the preceding sentence by pressing one of two buttons with their left and right index fingers. After fMRI sessions on Days 2, 3, and 9 participants received a pen and paper questionnaire with all 46 Alienese verbs in a random order. They were asked to translate these verbs into Dutch.

\section{FMRI experiment procedure}

The experiments were run using Presentation software (Neurobehavioral Systems, ). Participants lay in the scanner and looked at a screen via a mirror. On Day 1, a trial consisted of a white fixation cross on black background being displayed jittered between $400-3000 \mathrm{~ms}$, followed by a sentence for $2 \mathrm{~s}$. Sentences were presented in white "Arial" font of size 22 on a black background. On Day 2 and on prime trials on Days 3 and 9 (Fig. $1 a$ shows trial structures and timing), sentences were followed by a black blank screen jittered between 100-2100 ms and a picture for $3 \mathrm{~s}$. During target trials (Fig. 1a) on Days 3 and 9, two pictures instead of one were presented simultaneously for $4 \mathrm{~s}$ and the subject made a button press with his or her left or right index finger to choose between the left and the right picture.

\section{Behavioral analysis}

For the behavioral analysis, we analyzed the response choices using mixed-effects logit models (Pinheiro and Bates, 2000; Jaeger, 2008; Barr et al., 2013) with random effects for subjects and items in R (R Development Core Team, 2014). We followed the advice by Barr et al. (2013) and used a model with the maximal effect structure that was still converging. When a model did not converge, we removed random slopes for items before random slopes for subjects (since the variance for items is usually smaller) and interaction terms were removed before main effects. For contrast specifications deviation coding was used (comparing each level of a factor to the grand mean).

The model for the response choices included fixed effects for "Day" (Days 3, 9), "Type of Sentence" (Frequent, Infrequent, Known), "Verb" (Verb Repeated, Not Repeated), and "Syntax" (Syntax Repeated, Not Repeated) and allowed interactions between all these factors. The random effects structure included a random intercept for subjects and items, and random slopes for Syntax and Verb for subjects (this is the maximal random effect structure for which convergence is reached). For one subject the button presses were not registered on Day 3, so we excluded the subject from this analysis.

To assess the verb translation proficiency, we analyzed the number of correctly translated verbs out of the 46 items of the translation task after each day. We assessed whether there was a steady improvement over days, by using mixed-effects logit models (Pinheiro and Bates, 2000; Jaeger, 2008; Barr et al., 2013) with random effects for subjects and items in R (R Development Core Team, 2014) and a fixed effect for Day (Days $2,3,9)$. This is the maximal random effect structure for which convergence is reached.

\section{FMRI data acquisition}

Participants were scanned on a Siemens 3T Tim-Trio MRI-scanner, using a 32-channel coil. To acquire functional data we used parallelacquired inhomogeneity-desensitized fMRI (Poser et al., 2006; Buur et al., 2009). This is a multi-echo EPI sequence, in which images are acquired at multiple TEs following a single excitation ( $\mathrm{TR}=2.398 \mathrm{~s}$; each volume consisted of 31 slices of $3 \mathrm{~mm}$ thickness with slice-gap of $17 \%$; isotropic voxel size $=3.5 \times 3.5 \times 3 \mathrm{~mm}^{3}$; field-of-view $=224 \mathrm{~mm}$ ). The functional images were acquired at the following TEs: TE1 at $9.4 \mathrm{~ms}$, TE2 at $21.2 \mathrm{~ms}$, TE3 at $33 \mathrm{~ms}$, TE4 at $45 \mathrm{~ms}$, and TE5 at $56 \mathrm{~ms}$, with echo spacing of $0.5 \mathrm{~ms}$. This entails a broadened $\mathrm{T} 2 *$ coverage, because $\mathrm{T} 2 *$ mixes into the five echoes in different ways, and the estimate of $\mathrm{T} 2^{\star}$ is improved. The slices were acquired in an ascending order. In some subjects, parts of the top of the brain were outside the field-of-view. We made sure that most of the brain especially inferior parts of the frontal and temporal cortex were included. The anatomical images were acquired using a $\mathrm{T} 1$ weighted sequence.

\section{FMRI pre-processing}

The pre-processing as well as the first level analysis of the fMRI data were conducted with SPM8, second level analyses with SPM12 (). The first five functional images were discarded to ensure that transient non-saturation effects did not affect the analysis. The functional images (for each echo separately) were checked for spikes and if any were detected these images were removed and a replacement image was created based on the surrounding images. Spikes were detected in seven subjects; in six of these $<10$ spikes were found over all runs and echoes ( $<0.1 \%$ of the images); in one subject 67 images contained spikes (in $0.26 \%$ of the images). The images of the first echo were realigned to the subject-specific mean functional image and the realignment parameters were then copied to the other echoes. The five echoes were combined into one image using a method designed to filter task-correlated motion out of the signal (Buur et al., 2009). Subsequently, the functional images were slice-time corrected. The mean functional image was coregistered to the subjects' anatomical $\mathrm{T} 1$ image. The anatomical $\mathrm{T} 1$ images were then segmented into gray and white matter and the spatial normalization parameters were used to normalize the functional images. Finally, the functional images were smoothed with a $10 \mathrm{~mm}$ FWHM Gaussian kernel.

\section{First-level single-subject model}

The experiment consisted of a short sentence reading session on Day 1 (ie, before the learning sessions, the sentences were thus like strings of pseudowords to the participants), one session on Day 2 and two sessions each on Days 3 and 9. One subject took part in only one session on Day 3 and another in only one session on Day 9. However, despite less exposure to the language these participants showed a high level of proficiency and were thus kept in the analysis (they could translate $96 \%$ and $91 \%$ of the verbs on Day 9 and performed at $86 \%$ and $76 \%$ correct on the picture choice task on Day 9). Also, due to time constraints, the scan had to be stopped early on Day 2 on a couple of occasions; however, this resulted in the loss of $<5 \%$ of trials, randomly distributed across conditions.

For the first day, we modeled sentences and fixation cross intervals with one regressor each. For the subsequent days, within each session, the model for each individual subject included regressors that modeled the target sentences for the following conditions: syntactic repetition and verb repetition; syntactic repetition and no verb repetition; no syntactic repetition and verb repetition, as well as no syntactic repetition and no verb repetition, each of these for each type of sentence structure separately. The sentences were modeled from the start of their presentation. Further, we used one regressor for all prime sentences, for all intransitive sentences, all pictures, and fixation crosses (per session), respectively. The actual presentation time of an event was taken as its duration. All experimental regressors were convolved with a canonical hemodynamic response function. The realignment parameters for movement correction were also included in the model. Contrast images of the different repetition conditions were defined that were then taken to the second level for a random effects group analysis.

\section{Region-of-interest analysis}

Our main question concerned the processing of syntax within the artificial language. More specifically, we were interested in the difference between syntactic processing of novel versus known structures and its interaction with frequency within the syntactic processing network. To specifically test this, we conducted a region-of-interest analysis to test the effect of syntactic repetition, as well as the interaction of type of target structure (frequent, infrequent, known) and syntactic repetition. To define the core regions of the syntactic processing network we took the inverse inference activations to the query "syntactic" from the neurosynth meta-analysis tool () that exceeded a $Z$-value of 9 . The two resulting regions (see Fig. 3A) were located in LIFG and MTG/STG which coincide with the core regions that show syntactic repetition effects to familiar structures (Menenti et al., 2011; Segaert et al., 2012). Mean activations for the different syntactic repetition conditions (syntax re- 


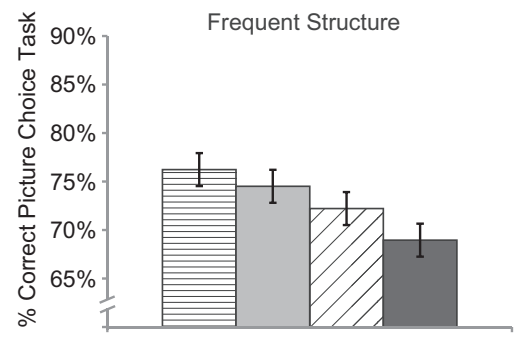

目 Verb Repeated, Syntax Repeated

$\square$ Verb Repeated, Syntax Not Repeated
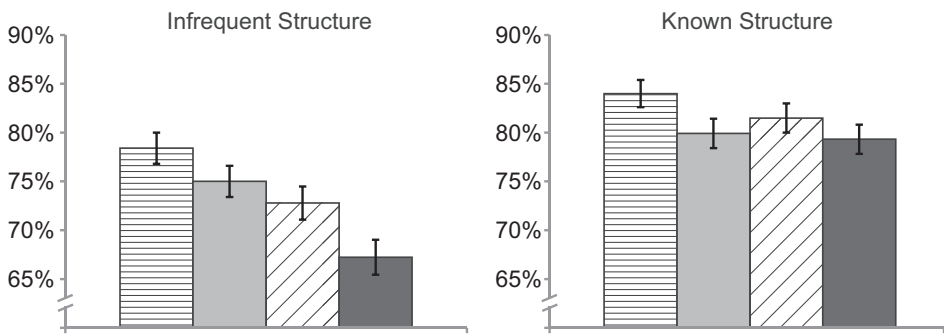

$\square$ Verb Not Repeated, Syntax Repeated

$\square$ Verb Not Repeated, Syntax Not Repeated

Figure 2. Behavioral results of the picture choice task displaying percentage correct picture choices per type of structure. Error bars indicate SEM.

peated frequent structure-syntax not repeated frequent structure; syntax repeated infrequent structure-syntax not repeated infrequent structure; syntax repeated known structure-syntax not repeated known structure) per region-of-interest were extracted using MarsBar () and entered into an ANOVA with the factors "Region" (LIFG, left posterior MTG/STG), "Day" (Days 3, 9), and "Type of Structure" (Frequent, Infrequent, Known) using SPSS 19.0.0. Next to the ANOVA looking at the main effects of syntactic repetition, as well as the interaction between type of structure and syntactic repetition in the two regions-of-interest, we also performed planned comparison one-sample $t$ tests to investigate whether the repetition effects per structure where larger than (for the novel structures) or smaller than zero (for the known structure). Furthermore, we investigated how the neural syntactic repetition effects are related to the learning process by looking at correlations with performance on the picture-choice task for these structures on the last day. As there was no significant difference in picture-choice task performance for infrequent and frequent structures, we pooled these conditions together, looking at the correlation with the neural syntactic repetition effect for novel structures. Because the performance on the known structures was significantly different from the novel structures, we performed a separate correlation of the performance on the known structures with the neural syntactic repetition effect for known structures. As the performance on the picture choice task is positively skewed, we used a logarithmic transform on the behavioral data and as we performed two correlations, we adjusted the $\alpha$ level to 0.025 .

\section{Second-level group analyses}

Moreover, we conducted whole-brain analyses to investigate the main effects of verb repetition and the interaction of verb repetition with syntactic repetition as well as day.

The main effect of verb repetition (averaged over Days 3 and 9). To test whether the main effect of verb repetition was significantly different from zero we used one-sample $t$ tests. We did not include Day 2 in these contrasts, as Day 2 was the initial learning session where the frequency of the different types of structure was different as well as the task.

Interaction between verb and syntactic repetition (averaged over Days 3 and 9). For the interaction between verb and syntactic repetition, we used a flexible factorial design with pooled error and correction for nonsphericity using ReML (Friston et al., 2002). The model was built on the syntactic repetition contrasts, included the factors "verb" (verb repetition or no verb repetition), and was designed to look at the interaction of verb and syntactic repetition. The model also included 20 participant effects.

Interaction between day (2, 3, and 9) and verb repetition. For the interaction between verb repetition and day, we used a flexible factorial design with pooled error and correction for nonsphericity using ReML (Friston et al., 2002). The model was built on the verb repetition contrasts, included the factor "day" (Days 2, 3, and 9) and was designed to look at the interaction between day and verb repetition. The model also included 20 participant effects.

All statistical parametric maps were thresholded at the voxel level at $p<0.001$ and cluster-level $p \mathrm{FWE}<0.0 .5$. All reported coordinates are in MNI space.
Relationship between language learning performance and the verb repetition effect. To investigate the relationship between the performance on the verb translation task on Day 9 (the learning outcome with regards to the "vocabulary") and the neural verb repetition effect, we tested for correlations between the verb repetition effects identified in point 1 and behavioral performance. To this end, we extracted the mean contrast values for each cluster using MarsBar () and correlated these with performance on the verb translation task on Day 9. As the performance on the verb translation task is positively skewed across participants, we used a logarithmic transform on the behavioral data.

\section{Results}

\section{Behavioral results}

\section{Picture responses}

There was a main effect of day, with better performance on Day $9(81 \%$ correct, SEM: $1 \%$ ) compared with Day 3 (71\%, SEM:1\%), $Z=-8.8, p<$ 0.001 . Moreover, verb repetition [verb repeated: $78 \%$ correct (SEM:1\%); verb not repeated: $74 \%$ correct (SEM:1\%)], as well as syntactic repetition [syntax repeated: 78\% correct (SEM: $1 \%$ ), syntax not repeated: 74\% correct (SEM:1\%)] helped the subjects in making the correct decision, $Z=-4.4$, $p<0.001$ and $Z=-2.4, p=0.02$, respectively, see Figure 2 . There was also a main effect of type (frequent, infrequent, known), as the performance on the known structure $[81 \%$ correct $(\mathrm{SEM}=1 \%)]$ was better than on the frequent $[73 \%$ correct $(\mathrm{SEM}=1 \%)], Z=-4.63, p<0.001$, or the infrequent structure $[73 \%$ correct $(\mathrm{SEM}=1 \%)], Z=-3.9, p<0.001$. The performance on the frequent and on the infrequent structure were not significantly different from each other $(Z<|1|)$. The syntactic priming effect did not interact with the type of structure $(Z<|1|)$.

\section{Verb translation}

There was a steady increase in the number of verbs that could be translated from Alienese into Dutch. Participants improved in translation performance from Day 2 to Day $3(Z=19.02, p<0.001)$ and from Day 3 to Day $9(Z=16.32, p<0.001)$. On Day 2, on average $15.54 \%$ of the verbs were translated (range: $0-65 \%$ ), on Day 3 this increased to $43.91 \%$ (range: $2-91 \%$ ) and further to $56.84 \%$ on Day 9 (range: $4-100 \%$ ).

\section{Neuroimaging results}

\section{Region-of-interest results: syntactic repetition effects}

As hypothesized, the repetition of the known type of structure led to a repetition suppression effect, whereas the repetition of the infrequent novel structure led to repetition enhancement, with the repetition effect to frequent novel structures patterning in between. The interaction of type of structure with the syntactic repetition effect (over Days 3 and 9) in our two regions-of-interest, LIFG and left posterior MTG/STG (Fig. 3) was significant: $F_{(2,38)}=5.39, p=0.009, \eta^{2}=0.22$. This effect did not differ across the two regions or between days. The main effect of syntactic repetition was not significant nor was its interaction with the factor day. Follow-up tests were performed to investigate the nature of the interaction between type of structure and syntactic repetition. The repetition enhancement effect to the infrequent structure was significantly larger 


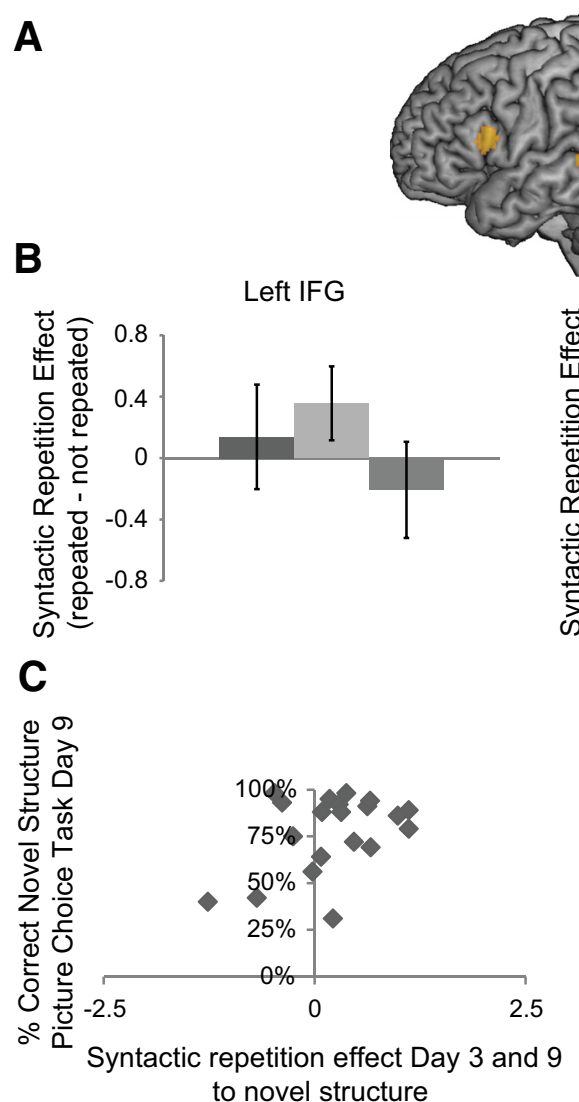

to novel structure
Left posterior MTG/STG
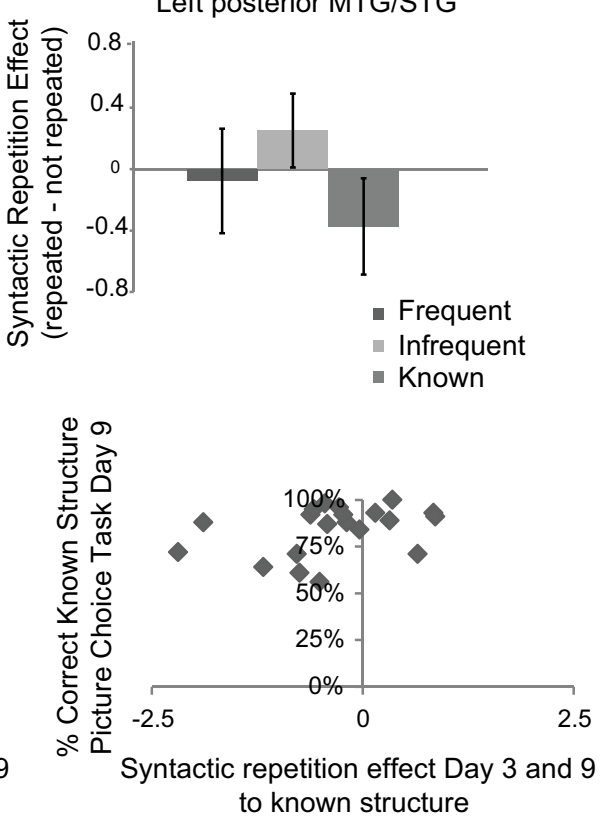

Figure 3. Results of the main region-of-interest analysis using Marsbar. $\boldsymbol{A}$, The two regions-of-interest in left inferior frontal and left posterior middle/superior temporal gyrus (defined using the activation maps to the query syntactic on the meta-analysis toolbox neurosynth.org thresholded at $Z>9$ ). $\boldsymbol{B}$, Mean contrast estimates for the syntactic repetition effects per type of structure in the two regions-of-interest averaged over Days 3 and 9. Error bars indicate SEM. C, Scatter plots showing the relationship between the neural syntactic repetition effects and behavioral performance. The left graph shows the relationship between the syntactic repetition effect to novel structures and the performance on the picture choice task on these structures on the last day of learning. The right graph illustrates the relationship between the syntactic repetition effect to the known syntactic structure and the performance on the picture choice task for this structure on the last day.

than the repetition effect to the known structure: $t_{(19)}=3.2, p=0.006$. Similarly, the repetition enhancement effect to the frequent structure was also significantly larger than the repetition effect to the known structure: $t_{(19)}=1.8, p=0.045$. Although the trend goes in the right direction, the repetition enhancement effect to the infrequent structure was not significantly larger than the repetition enhancement effect to the frequent structure: $t_{(19)}=1.6, p=0.066$.

Planned comparisons were performed to test whether the repetition enhancement effects to frequent and infrequent structures were larger and the repetition suppression effect to known structures significantly smaller than zero. The repetition effect to frequent structures was not significantly different from zero: $t_{(19)}=0.26, p=0.8$; in contrast, the repetition enhancement effect to infrequent structures was significantly larger than zero: $t_{(19)}=2.43, p=0.0125$, whereas the syntactic repetition suppression effect to known structures was significantly $<0: t_{(19)}=$ $-1.94, p=0.034$.

The relationship between the syntactic repetition enhancement effect to novel syntactic structures (across both regions and days) and the performance on the picture choice task on Day 9 for these structures revealed a significant positive correlation: $r=0.45, p=0.023$, whereas the correlation between the syntactic repetition effect to known structures and the performance on the picture choice task on Day 9 for known structures was not significant: $r=0.37, p=0.054$ (Fig. 3).

Whole brain: verb repetition effect

Over Days 3 and 9, verb repetition resulted in repetition enhancement effects in a wide-spread network of left and right temporal regions ex- tending into parietal areas, cingulate cortex, as well as the right inferior frontal gyrus, the precuneus, and other occipital regions (Fig. $4 a$; Table 2). In a subset of these regions, mainly the precuneus and the right middle temporal gyrus extending into inferior parietal regions, the repetition enhancement effects increased from Day 2 to Day 9 (Fig. $4 b$; Table 2). To test whether the strength of the verb repetition enhancement effect increased with proficiency on the verb task, we correlated the verb repetition enhancement effect in each of the five clusters with the performance on the verb translation task on Day 9. As we tested five correlations, we set the $\alpha$ level to 0.01 . The clusters in left parietal and right temporal/parietal cortex showed a trend toward a positive correlation between the verb repetition enhancement effect and the performance on the verb translation task on Day 9: $r=0.38, p=0.051$ and $r=0.41, p=$ 0.036 , respectively. The other clusters did not show a trend toward a correlation, all $r<|.2|$.

Whole brain: interactions between verb and syntax repetition

Interactions between verb and syntactic repetition were found in left angular gyrus, extending slightly into the temporal cortex (Fig. 4c). These interactions were driven by a stronger RE effect if both verb and syntax were repeated.

\section{Discussion}

In this fMRI repetition study, participants implicitly learned words and syntactic structures of an artificial miniature language over several days. The syntactic structures were chosen such that one corresponded to a familiar structure of the native Dutch language and two others did not. The two novel structures occurred with different frequencies in the first training session (Day 2). Participants were able to learn the words and syntactic structures over the course of the experiment. Behaviorally, we found structural repetition effects on the picture choice task that did not differ between syntactic structures. However, overall, participants performed better on the familiar structure. Moreover, verb repetition helped in making a correct decision.

Both the LIFG and the left posterior MTG/STG (ROI analysis; Fig. 3), regions known to be involved in syntactic processing, showed a dissociation between fMRI repetition effects: showing RS to familiar structures and RE to infrequent unfamiliar structures. Verb and word order repetition interacted in left angular gyrus, indicating a lexical boost to the syntactic repetition effect. Verb repetition lead to RE in the left and right posterior temporal and inferior parietal regions. Parts of the verb RE effects increased continuously over days (Fig. 4). The behavioral learning outcome and the RE effect to unfamiliar structures are correlated; there was a hint of a similar effect between the verb RE effect and the number of verbs learned.

RS is a well known response to the repetition of syntactic structures in the first language and established ones in a second language (Weber and Indefrey, 2009; Menenti et al., 2011; Segaert et al., 2012). The observed RS effect for the familiar word order can thus be related to similar effects observed for syntactic repetition in studies using natural language and suggests that the 
known structure in the new language had been mapped onto its Dutch counterpart. The present result suggests that even when structural information is realized in a new (artificial) language, it appears to be integrated into the same neural structures as the native language, if there is sufficient structural overlap. That such a mapping for structures that are similar between languages is possible is supported by cross-linguistic syntactic repetition suppression effects (Weber and Indefrey, 2009). From a methodological perspective, this result strengthens the suggestion that artificial language learning paradigms can tap into the same underlying neural mechanisms as used for a natural language (Petersson and Hagoort, 2012).

Contrary to the repetition suppression effect to familiar structures, the repetition of unfamiliar structures (as well as novel words, see discussion below) led to repetition enhancement (Fig. 3). This pattern of effects ties in with similar dissociations that have been found to the repetition of pseudowords compared with words (Fiebach et al., 2005; Gagnepain et al., 2008), suggesting that the RE effects might be related to the building of new representations for these novel word orders. The infrequent novel structure was particularly sensitive to repetition (its RE effect was significantly different from zero and there was a trend toward a stronger effect compared with the frequent structures). This relates the magnitude of RE to the strength of a novel representation, given that the representations of the less frequently trained structure were arguably weaker. The repetition effect to the frequent structure was not significantly different from zero, which might mean that it is an effect halfway between RS and RE. We thus suggest that the RE effect reflects learning processes that strengthen the new representation being built, an effect that we predict will switch to RS once a stable memory representation has been established. The notion that the RE effect is related to the learning process is further strengthened by the observation that the strength of the enhancement effect correlates with learning progress. What exactly is represented or processed may depend on the cortical region involved. Whereas the left posterior middle/superior temporal gyrus has been linked to linguistic representations, such as stored lexical and syntactic information, the left inferior frontal gyrus has been linked to online processing. It is thought to unify syntactic building blocks during both language comprehension and production (Hagoort, 2005; Snijders et al., 2009; Hagoort and Indefrey, 2014). RE in left inferior frontal gyrus might, therefore, reflect a learning process in which repetition enables additional unification operations on the target, whereas the effect in left posterior middle/superior temporal gyrus might reflect the strengthening of the linguistic representation of the word order. Although we have interpreted the repetition effects as driven by distributional patterns of syntactic structure, ie, the order of grammatical roles (subject, object, verb), we cannot exclude that their mapping onto thematic roles (agents, patients, action) contributed to the observed effects.
It is possible to link the pattern of repetition effects to the implicit learning theory of syntactic priming (Chang et al., 2000), if one assumes that an improvement of a representation upon repetition may not only mean a "sharpening," requiring fewer neurons, as in the case of established representations, but also an expansion of the neuronal substrate in the case of new representations. Predictive coding theories (Friston, 2005) predict RS for familiar structures, because the amount of neural activation depends on the size of the prediction error, which becomes smaller with repetition of an identical structure. During the learning of an unfamiliar structure on the other hand, increases in the precision of prediction errors might initially lead to repetition enhancement (Auksztulewicz and Friston, 2016). These predictive coding effects during learning might lead to a U-shaped pattern of activations to novel stimuli (reflected in changes in repetition effects from enhancement to suppression) from "no learning" to "early learning" to "expertise" (Price and Devlin, 2011).

RE effects to repeated verbs were found in brain regions linked to lexical and semantic processing (Fig. 4), that are also seen in studies on word and semantic processing in the first language (Binder and Desai, 2011; Menenti et al., 2011; Price, 2012), as well as during language learning (Mestres-Missé et al., 2008; Davis et al., 2009), including regions in the middle temporal gyrus. This verb RE effect is in a location slightly more inferior to the left posterior MTG/STG ROI showing syntactic repetition effects. The verb RE effects are consistent with accounts connecting RE 
Table 2. Whole-brain repetition effects

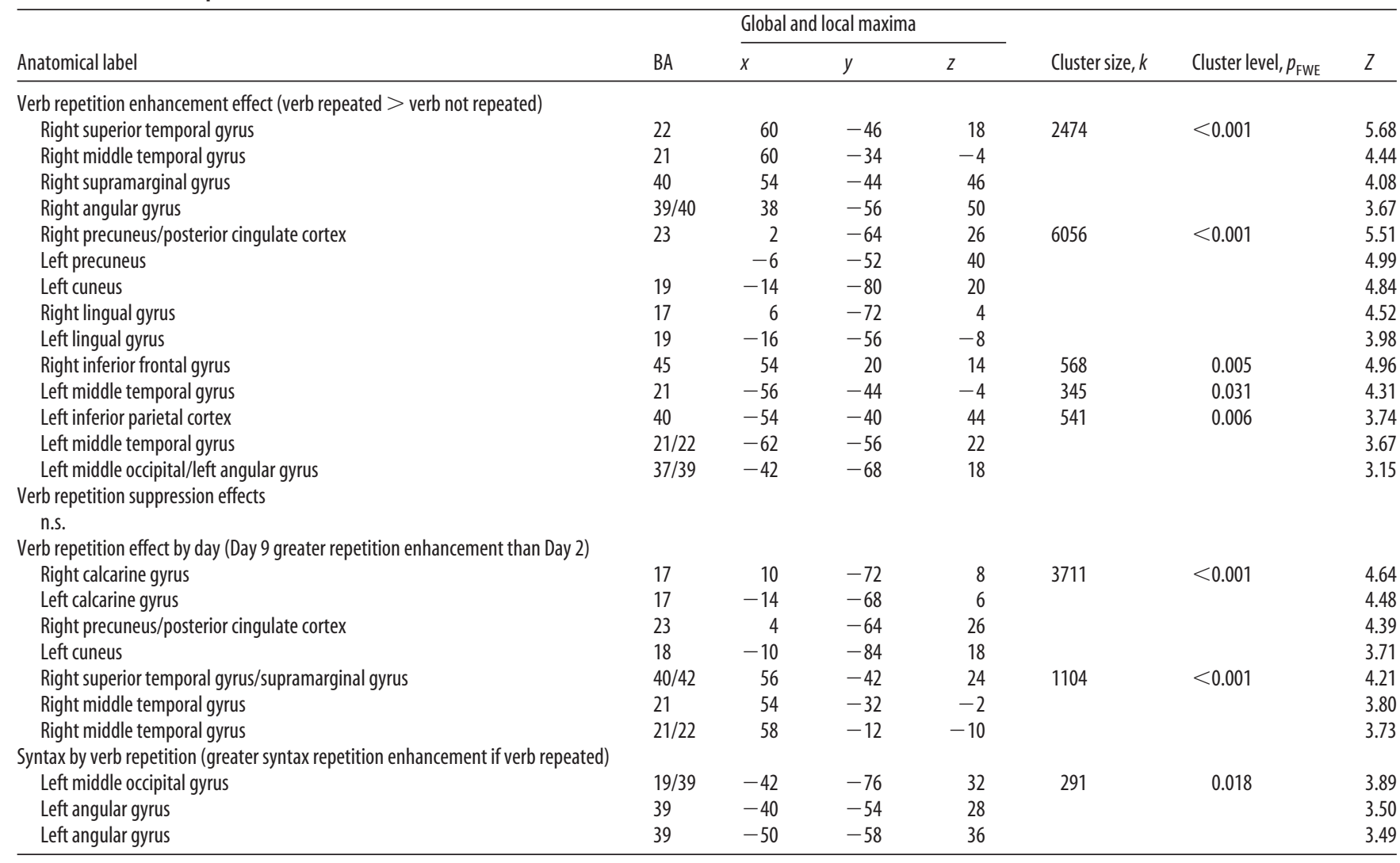

effects to the built-up of novel representations, in the present case novel words with rich semantic information attached. The observed RE effect might reflect the gradual strengthening of a lexical-semantic mapping. Interestingly, most of these RE effects increased over the course of the different days. This further supports the idea that RE effects might be linked to language learning, reflecting a steady build-up of these new lexical-semantic representations.

Moreover, verb repetition boosted the syntactic RE effect in the left angular gyrus (also present in the right hemisphere homolog but this did not survive cluster-level correction; Fig. 4). This interaction provides evidence that verb-specific, lexically driven syntactic processing effects might be found early on during learning that would be compatible with proposals of a lexical nature of syntactic processing (Vosse and Kempen, 2000; Jackendoff, 2002; Snijders et al., 2009; Christiansen and Chater, 2015). Of note should be, however, that we also find main effects of syntactic repetition independent of verb repetition both at the behavioral and neural (in the ROI analysis) level. Some lexically driven but also some lexically independent syntactic repetition effects were also found in a behavioral-only version of the present experiment (Weber, 2012). Thus, although lexical information is important during syntactic processing, abstract syntactic processing effects can be found very early on during learning.

The region showing the interaction between the verb and the syntactic repetition effect, the angular gyrus, has been linked to semantic representations independent of modality (Binder and Desai, 2011), and even more relevant to effects of combining concepts into larger meaning representations (Price et al., 2015). This could be linked to theories in memory research that talk about neocortical schema representations (Tse et al., 2007) that are due to the establishment of an abstract pattern, in this case a pattern that links lexical-semantic and syntactic information/ regularities. Thus, when the verb and the thematic roles are repeated, a larger combined structured meaning representation may be primed.

The steadily increasing repetition enhancement effects to verbs, even after days, speaks for a longer time frame for these types of linguistic information to become stabilized in the more complex environment of an artificial language compared with other learning effects that merely require overnight consolidation (Walker and Stickgold, 2006; Davis et al., 2009; Nieuwenhuis et al., 2013). We do expect a shift from RE to RS once memory representations have stabilized. This hypothesis should be investigated with a longitudinal study of syntactic and verb repetition effects with a longer time frame and more fine-grained behavioral measures of the state of learning progress.

\section{Conclusion}

In conclusion, the dissociation between RE for unfamiliar grammatical structures and RS for familiar ones, suggests that repetition effects reflect a neural learning mechanism. A similar pattern of effects for verb learning shows that repetition effects are indicative of a general mechanism for building or strengthening novel neural representations.

\section{References}

Auksztulewicz R, Friston K (2016) Repetition suppression and its contextual determinants in predictive coding. Cortex. Advance online publication. Retrieved March 22, 2016. doi:10.1016/j.cortex.2015.11.024.

Barr DJ, Levy R, Scheepers C, Tily HJ (2013) Random effects structure for confirmatory hypothesis testing: keep it maximal. J Mem Lang 68: 255-278. CrossRef

Binder JR, Desai RH (2011) The neurobiology of semantic memory. Trends Cogn Sci 15:527-536. CrossRef Medline 
Buur PF, Poser BA, Norris DG (2009) A dual echo approach to removing motion artefacts in fMRI time series. NMR Biomed 22:551-560. CrossRef Medline

Chang F, Dell GS, Bock K, Griffin ZM (2000) Structural priming as implicit learning: a comparison of models of sentence production. J Psycholinguist Res 29:217-229. CrossRef Medline

Christiansen MH, Chater N (2015) The now-or-never bottleneck: a fundamental constraint on language. Behav Brain Sci. Advance online publication. Retrieved March 22, 2016. doi:10.1017/S0140525X1500031X.

Davis MH, Di Betta AM, Macdonald MJ, Gaskell MG (2009) Learning and consolidation of novel spoken words. J Cogn Neurosci 21:803-820. CrossRef Medline

Ferreira VS, Bock K (2006) The functions of structural priming. Lang Cogn Process 21:1011-1029. CrossRef Medline

Fiebach CJ, Gruber T, Supp GG (2005) Neuronal mechanisms of repetition priming in occipitotemporal cortex: spatiotemporal evidence from functional magnetic resonance imaging and electroencephalography. J Neurosci 25:3414-3422. CrossRef Medline

Friederici AD, Gierhan SM (2013) The language network. Curr Opin Neurobiol 23:250-254. CrossRef Medline

Friston K (2005) A theory of cortical responses. Philos Trans R Soc Lond B Biol Sci 360:815-836. CrossRef Medline

Friston KJ, Penny W, Phillips C, Kiebel S, Hinton G, Ashburner J (2002) Classical and Bayesian inference in neuroimaging: theory. Neuroimage 16:465-483. CrossRef Medline

Gagnepain P, Chételat G, Landeau B, Dayan J, Eustache F, Lebreton K (2008) Spoken word memory traces within the human auditory cortex revealed by repetition priming and functional magnetic resonance imaging. J Neurosci 28:5281-5289. CrossRef Medline

Grill-Spector K, Henson R, Martin A (2006) Repetition and the brain: neural models of stimulus-specific effects. Trends Cogn Sci 10:14-23. CrossRef Medline

Hagoort P (2005) On Broca, brain, and binding: a new framework. Trends Cogn Sci 9:416-423. CrossRef Medline

Hagoort P, Indefrey P (2014) The neurobiology of language beyond single words. Annu Rev Neurosci 37:347-362. CrossRef Medline

Henson RN, Rugg MD (2003) Neural response suppression, haemodynamic repetition effects, and behavioural priming. Neuropsychologia 41: 263-270. CrossRef Medline

Henson R, Shallice T, Dolan R (2000) Neuroimaging evidence for dissociable forms of repetition priming. Science 287:1269-1272. CrossRef Medline

Jackendoff R (2002) Foundations of language: brain, meaning, grammar, evolution. New York: Oxford UP.

Jaeger TF (2008) Categorical data analysis: away from ANOVAs (transformation or not) and towards logit mixed models. J Mem Lang 59:434-446. CrossRef Medline

Menenti L, Gierhan SM, Segaert K, Hagoort P (2011) Shared language: overlap and segregation of the neuronal infrastructure for speaking and listening revealed by functional MRI. Psychol Sci 22:1173-1182. CrossRef Medline

Mestres-Missé A, Càmara E, Rodriguez-Fornells A, Rotte M, Münte TF (2008) Functional neuroanatomy of meaning acquisition from context. J Cogn Neurosci 20:2153-2166. CrossRef Medline

Nieuwenhuis IL, Folia V, Forkstam C, Jensen O, Petersson KM (2013) Sleep promotes the extraction of grammatical rules. PLoS One 8:e65046. CrossRef Medline

Opitz B, Friederici AD (2004) Brain correlates of language learning: the neuronal dissociation of rule-based versus similarity-based learning. J Neurosci 24:8436-8440. CrossRef Medline

Petersson KM, Hagoort P (2012) The neurobiology of syntax: beyond string sets. Philos Trans R Soc Lond B Biol Sci 367:1971-1983. CrossRef Medline

Petersson KM, Forkstam C, Ingvar M (2004) Artificial syntactic violations activate Broca's region. Cogn Sci 28:383-407. CrossRef

Pickering MJ, Branigan HP (1998) The representation of verbs: evidence from syntactic priming in language production. J Mem Lang 39:633-651. CrossRef

Pinheiro JC, Bates DM (2000) Linear mixed-effects models: basic concepts and examples. In: Mixed-effects models in S, S-PLUS. New York: Springer.

Poser BA, Versluis MJ, Hoogduin JM, Norris DG (2006) BOLD contrast sensitivity enhancement and artifact reduction with multiecho EPI: parallel-acquired inhomogeneity-desensitized fMRI. Magn Reson Med 55:1227-1235. CrossRef Medline

Price AR, Bonner MF, Peelle JE, Grossman M (2015) Converging evidence for the neuroanatomic basis of combinatorial semantics in the angular gyrus. J Neurosci 35:3276-3284. CrossRef Medline

Price CJ (2012) A review and synthesis of the first 20 years of PET and fMRI studies of heard speech, spoken language and reading. Neuroimage 62 : 816-847. CrossRef Medline

Price CJ, Devlin JT (2011) The interactive account of ventral occipitotemporal contributions to reading. Trends Cogn Sci 15:246-253. CrossRef Medline

R Core Team (2014) R: A language and environment for statistical computing. $\mathrm{R}$ Foundation for Statistical Computing, Vienna, Austria. http://www.R-project.org/.

Segaert K, Menenti L, Weber K, Petersson KM, Hagoort P (2012) Shared syntax in language production and language comprehension: an fMRI study. Cereb Cortex 22:1662-1670. CrossRef Medline

Segaert K, Weber K, de Lange FP, Petersson KM, Hagoort P (2013) The suppression of repetition enhancement: a review of fMRI studies. Neuropsychologia 51:59-66. CrossRef Medline

Snijders TM, Vosse T, Kempen G, Van Berkum JJ, Petersson KM, Hagoort P (2009) Retrieval and unification of syntactic structure in sentence comprehension: an fMRI study using word-category ambiguity. Cereb Cortex 19:1493-1503. CrossRef Medline

Tooley KM, Bock K (2014) On the parity of structural persistence in language production and comprehension. Cognition 132:101-136. CrossRef Medline

Tse D, Langston RF, Kakeyama M, Bethus I, Spooner PA, Wood ER, Witter MP, Morris RG (2007) Schemas and memory consolidation. Science 316:76-82. CrossRef Medline

Vosse T, Kempen G (2000) Syntactic structure assembly in human parsing: a computational model based on competitive inhibition and a lexicalist grammar. Cognition 75:105-143. CrossRef Medline

Walker MP, Stickgold R (2006) Sleep, memory, and plasticity. Annu Rev Psychol 57:139-166. CrossRef Medline

Weber K (2012) The language learning brain: evidence from second language and bilingual studies of syntactic processing. Nijmegen, The Netherlands: Radboud University Nijmegen.

Weber K, Indefrey P (2009) Syntactic priming in German-English bilinguals during sentence comprehension. Neuroimage 46:1164-1172. CrossRef Medline 